\title{
Antibiotic Stewardship: An Important Pharmacy Role?
}

\author{
Christine M Bond
}

M any thousands of people die each year from infections caused by antibiotic-resistant bacteria. The increasing incidence of antibiotic resistance has been described as a global public health threat, and concerted efforts are required to address the problem. In this issue of the CJHP, Wu and others ${ }^{1}$ describe a small audit of antibiotic use in a long-term care facility. The audit identified overdependence on third-generation cephalosporins and fluoroquinolones, together with presumptive diagnoses in the absence of sensitivity testing. Although describing a small audit in just one facility, the paper illustrates that, despite widespread recognition of the problem of antibiotic resistance, there remains a disconnect between knowledge and current practice.

Antibiotics have been central to improved population health since they were first introduced as a treatment in the 1940s. Indeed, their dramatic effect on the course of infectious diseases was said to "close the book on infectious diseases". ${ }^{2}$ However, we now know that this is not the case. The increasing use of antibiotics in medicine has led to overdependence on the part of patients and overprescribing by professionals. These issues, together with the use of antibiotics in animal husbandry, have resulted in the development of antibiotic resistance and a consequent reduction in the effectiveness of the current armamentarium of anti-infective treatments. At the same time, the rate of development of new antibiotics has slowed, failing to compensate for the effective removal of existing treatments from the dispensary shelf. Indeed, only 2 new classes of antibiotics (oxazolidinones and cyclic lipopeptides) have been brought to market in the past 30 years. ${ }^{3}$ The reasons for this paucity of new antibiotics include the many challenges in today's drug development pipeline. Examples are the increasing time it takes to bring a new drug to market, the costly attrition that occurs during the process, and the overall financial investment required. Furthermore, in contrast to the situation for drugs used to treat chronic conditions, the return on investment for antibiotics is unattractive, and the commercial viability of any new antibiotic is poor. In general, antibiotics are taken for short- term treatment and are largely curative. Additionally, any new antibiotic that is developed will almost certainly be accompanied by guidance limiting its use unless all other treatments have failed. So, although novel approaches to identifying new antibiotics are being explored, such as identifying new sources of

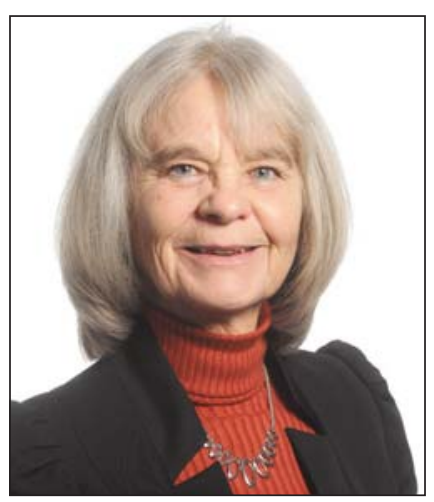
molecules from the plant and animal kingdoms and investigating new mechanisms of action (including the use of bacteriophages and gene-specific antibodies ${ }^{4}$ ), these can be, at best, only longterm solutions.

In the absence of new antibiotics for the foreseeable future, it is incumbent on the public and on health care professionals to take care of the antibiotics we already have through what is known as antibiotic stewardship (or antimicrobial stewardship). This term encompasses a range of activities, such as better hygiene and handwashing regimens, as well as appropriate prescribing (i.e., prescribing an antibiotic only when necessary and, if such a drug is needed, making sure it is the right one at the right dose, administered for the right duration). At the organizational level, antibiotic stewardship translates into systems of monitoring and guidance to promote evidence-based use. ${ }^{5}$ At the patient level, antibiotic stewardship is the "optimal selection, dosage and duration of treatment that results in the best clinical outcome for the treatment or prevention of infection, with minimal toxicity to the patient and minimal impact on subsequent resistance". 6

It should be well known by now-to professionals if not the wider public - that many infections, and not just viral infections, do not need treatment with an antibiotic. For example, evidence supports the use of symptomatic treatment rather than an anti- 
biotic for self-limiting conditions such as otitis media, since use of an antibiotic for such indications does little to reduce the duration of the associated symptoms. ${ }^{7}$ Indeed, it is the overuse of antibiotics in primary care that has led to the development of antibiotic resistance in the community, which often remains silent until the patient is admitted to hospital or a long-term care facility.

It is generally accepted that prescribers do not always adhere to evidence-based practice. Instead, they revert to treating the individual rather than considering the longer-term impact of the treatment on that patient (once antibiotic resistance has developed in an individual, it can last for over a year ${ }^{8}$ ) or the population. Pharmacists are generally one step removed from prescribing antibiotics to patients and can therefore counsel prescribers with objective professional advice. As experts in medicines, pharmacists are well placed to lead the antibiotic stewardship initiative, not just by doing audits and highlighting the problem but by influencing those crucial prescribing decisions. In fact, the role of the pharmacist on antibiotic stewardship teams has already been advocated. ${ }^{9-11}$ Sadly, however, there has been limited research in this area, and this role for pharmacists is not underpinned by good empirical evidence.

The systematic review by Kaki and others ${ }^{12}$ (referenced by Wu and others $\left.{ }^{1}\right)$ is limited in its generalizability, as it included only papers conducted in critical care. However, it did show that antibiotic stewardship could lead to reductions in antibiotic utilization and costs, shorter duration of treatment, more appropriate use of these drugs, fewer adverse events, and less long-term use. Significantly, antibiotic stewardship was found not to lead to increases in nosocomial infections, length of stay, or mortality. However, the authors of the review made no mention of the role of pharmacists in any of the interventions described.

A recent $\mathrm{UK}$ study ${ }^{13}$ has provided encouraging data on the positive effect of a pharmacist-led consultant audit and feedback program for improving the quality of antibiotic prescribing over an 18-month period, specifically by improving adherence to guidelines and recording an indication for use in the medical record. Despite the limitations of this single-site study with a before-and-after design, this report confirms my belief that pharmacists have a future major role to play in combatting the global threat we all face. Similar anecdotal reports confirm this belief, ${ }^{2}$ but we need more evidence from randomized controlled trials to make the case for antibiotic stewardship to become a mainstream role for pharmacists. Whether you are working in primary care, intermediate care, secondary care, tertiary care, or long-term care, please help in generating that evidence!
References

1. Wu LDY, Walker SAN, Elligsen M, Palmay L, Simor A, Daneman N. Antibiotic use and need for antimicrobial stewardship in long-term care. Can J Hosp Pharm. 2015;68(6):445-9.

2. Upshur R. Ethics and infectious disease [book review]. Bull World Health Organ. 2008;86(8):654. Also available from: www.who.int/bulletin/ volumes/86/8/08-056242/en/

3. Coates A, Halls G, Hu Y. Novel classes of antibiotics or more of the same? Br J Pharmacol. 2011;163(1):184-94.

4. New medicines, better medicines, better use of medicines: a guide to the science underpinning pharmaceutical practice. London (UK): Royal Pharmaceutical Society; 2014 [cited 2015 Sep 28]. Available from: www.rpharms.com/ promoting-pharmacy-pdfs/nmbmbu---full-report.pdf

5. Tamma PD, Cosgrove SE. Antimicrobial stewardship. Infect Dis Clin North Am. 2011;25(1):245-60.

6. Gerding DN. The search for good antimicrobial stewardship. Jt Comm J Qual Improve. 2001;27(8):403-4.

7. Little P, Gould C, Moore M, Warner G, Dunleavy J, Williamson I. Predictors of poor outcome and benefits from antibiotics in children with acute otitis media: pragmatic randomised trial. BMJ. 2002;325(7354):22.

8. Costelloe C, Metcalfe C, Lovering A, Mant D, Hay A. Effect of antibiotic prescribing in primary care on antimicrobial resistance in individual patients: systematic review and meta-analysis. BMJ. 2010;340:c2096.

9. Dellit TH, Owens RC, McGowan JE Jr, Gerding DN, Weinstein RA, Burke JP, et al. Infectious Diseases Society of America and the Society for Healthcare Epidemiology of America guidelines for developing an institutional program to enhance antimicrobial stewardship. Clin Infect Dis. 2007;44(2):159-77.

10. Antimicrobial stewardship: statement on the role of the pharmacist. Ottawa (ON): Canadian Society of Hospital Pharmacists; 2014 [cited 2015 Sep 30]. Available from: www.cshp.ca/dms/dmsView/1_AntimicrobialStewardship-Statement-FINAL.pdf

11. ASHP statement on the pharmacist's role in antimicrobial stewardship and infection prevention and control. Bethesda (MD): American Society of Health-System Pharmacists; [cited 2015 Sep 30]. Available from: www.ashp.org/doclibrary/bestpractices/specificstantimicrob.aspx

12. Kaki R, Elligsen M, Walker S, Simor A, Palmay L, Daneman N. Impact of antimicrobial stewardship in critical care: a systematic review. J Antimicrob Chemother. 2011;66(6):1223-30.

13. Roberts E, Dawoud DM, Hughes DA, Cefai C. Evaluation of a consultant audit and feedback programme to improve the quality of antimicrobial prescribing in acute medical admissions. Int J Pharm Pract. 2015;23(5):333-9.

Christine M Bond, BPharm, PhD, MEd, is with the Pharmacy, Centre of Academic Primary Care, University of Aberdeen, Foresterhill, Aberdeen, Scotland. She is also an Associate Editor with the CJHP.

Competing interests: Christine Bond is Editor in Chief of the International Journal of Pharmacy Practice and is a member of the Editorial Boards for the Journal of Self Care, Prescriber, Pilot and Feasibility Studies, and the Journal of Pharmaceutical Health Services Research. She also chairs the Expert Advisory Panel for Science of the Royal Pharmaceutical Society.

Address correspondence to:

Professor Christine M Bond

Pharmacy

Centre of Academic Primary Care

Room 1.123

Polwarth Building West Block

Foresterhill, Aberdeen AB25 2ZD

Scotland

e-mail : c.m.bond@abdn.ac.uk 